\title{
Efectividad Analgésica de Paracetamol Post-Cirugía Mucogingival: Ensayo Clínico Aleatorizado Paralelo
}

\author{
Analgesic Effectiveness of Paracetamol Post-Mucogingival Surgery: \\ Parallel Randomized Clinical Trial
}

Karen Mishell Burgos Quiróz'; Angel S. Asmat-Abanto ${ }^{1,2}$ \& Rosita Elena Espejo-Carrera ${ }^{1,3}$

BURGOS QUIRÓZ, K. M.; ASMAT-ABANTO, A. S. \& ESPEJO-CARRERA, R. E. Efectividad analgésica de paracetamol post-cirugía mucogingival: ensayo clínico aleatorizado paralelo. Int. J. Odontostomat., 13(2):184-188, 2019.

RESUMEN: El objetivo de este trabajo fue determinar la efectividad analgésica de paracetamol post-cirugía mucogingival. El estudio, prospectivo, longitudinal, comparativo y experimental, se desarrolló en la Clínica Odontológica de la Universidad Privada Antenor Orrego (Trujillo, Perú) con los pacientes de la asignatura Periodoncia II. Se incluyeron 39 pacientes, quienes fueron distribuidos de manera aleatoria en 2 grupos de 19 y 20 cada uno, según los criterios establecidos. La medicación se inició 20 minutos después de concluida la cirugía, y se mantuvo por 24 horas. Para determinar la efectividad analgésica se usó la escala visual analógica $(0-100 \mathrm{~mm})$ y se recogió la información que fue procesada, utilizando la prueba de U Mann-Whitney con un nivel de significancia del $5 \%$. No existe diferencia en cuanto a la efectividad analgésica post-cirugías mucogingivales entre paracetamol y el control a las 2 horas $(p=0.415), 8$ horas $(p=0.279)$ y 24 horas $(p=0.736)$. Paracetamol es efectivo como analgésico post-cirugía mucogingival.

PALABRAS CLAVE: efectividad, analgésicos, acetaminofén, periodontal, escala visual analógica.

\section{INTRODUCCIÓN}

La cirugía periodontal mucogingival es un procedimiento quirúrgico que resuelve procesos infecciosos y permite mantener la posición y función del periodonto (Godoy et al., 2014). Es uno de los tratamientos quirúrgicos más usados para la estética odontológica en la actualidad (Martínez Hernández \& Marín González, 2013).

La eliminación de las estructuras de soporte del diente provoca dolor postoperatorio debido a la respuesta inflamatoria (Zardo et al., 2013); por ello, presentar dolor y edema después de una cirugía periodontal son sucesos comunes (Peres et al., 2012).

El dolor después de una cirugía periodontal es una experiencia sensorial, emocional y compleja (Eli et al., 2000), altamente subjetiva, por lo tanto varía considerablemente entre los individuos (Hungund \&
Thakkar, 2011). Según Betancourt et al. (2004), el 70 $\%$ de pacientes percibe algún grado de dolor después de la cirugía periodontal, aunque la mayoría es de leve intensidad.

El dolor después de una cirugía periodontal puede alcanzar su punto máximo en las primeras 24 horas, y disminuir rápidamente en los días posteriores de la intervención quirúrgica (Moore et al., 2011; Durand et al., 2013).

Los profesionales de la salud deben brindar un tratamiento efectivo una vez culminado todo procedimiento quirúrgico (Pickett, 2012). Por lo tanto, es necesario evaluar los fármacos que proporcionen una adecuada efectividad analgésica y comodidad para el paciente, en los días posteriores de haber realizado la cirugía periodontal (Zardo et al.).

\footnotetext{
${ }^{1}$ Escuela Profesional de Estomatología de la Universidad Privada Antenor Orrego, Trujillo, Perú.

${ }^{2}$ Escuela de Postgrado de la Universidad Nacional de Trujillo, Perú.

${ }^{3}$ Maestría de Ciencias en Investigación Clínica, Escuela de Posgrado de la Univesidad Privada Antenor Orrego, Perú.
} 
Entre las opciones terapéuticas para el tratamiento del dolor post operatorio se encuentran los antiinflamatorios no esteroideos (AINE) (Ardila Medina, 2012). Este grupo de analgésicos es ampliamente utilizado para minimizar el dolor asociado con varias condiciones periodontales (Betancourt et al.). Los AINE inhiben la enzima ciclooxigenasa (COX), interfiriendo con la liberación de prostaglandinas (Hungund \& Thakkar), las cuales son las causantes de la inflamación y dolor en el sitio de la lesión (Jaiswal et al., 2014).

Paracetamol es un analgésico-antipirético usado clínicamente durante más de medio siglo (Ramachandran et al., 2012), y entre sus indicaciones están el dolor de cabeza, dolor dental y dolores musculares (Coulthard et al., 2014). Debido a sus mínimos efectos negativos es de primera elección para el alivio del dolor de leve a moderado (Zahrowski, 2010). Paracetamol no tiene un mecanismo de acción claramente definido, pero se acepta que actúa inhibiendo a la ciclooxigenasa $1(\mathrm{COX}-1)$ y la ciclooxigenasa 3 (COX-3), reduciendo la liberación de prostaglandinas en el sistema nervioso central (Gehling et al., 2010; Coulthard et al.).

Debido a la gran variedad de fármacos en el mercado y a la falta de estudios sobre analgésicos en cirugías mucogingivales, se realizó este trabajo que tuvo por finalidad determinar la efectividad analgésica de paracetamol post-cirugía mucogingival. Del mismo modo, se busca mejorar la atención de los pacientes, buscando su satisfacción post-operatoria, y contribuir a la generación de guías terapéuticas basadas en evidencia para realizar cirugías periodontales.

\section{MATERIAL Y MÉTODO}

El presente fue un ensayo clínico en fase IV, de diseño paralelo aleatorizado y ciego simple, que se realizó en la Clínica Odontológica de la Universidad Privada Antenor Orrego (Trujillo, Perú), en los turnos de práctica clínica de la asignatura de Periodoncia II del programa de Estomatología, en noviembre de 2016. Para determinar el tamaño muestral se usaron datos del estudio de Rashwan (2009), empleándose la fórmula para comparación de promedios cuando la población es indefinida o infinita, bajo los parámetros siguientes: $\alpha=0,1$ (máximo error tipo I), $1-\alpha / 2=0,95$ (nivel de confianza de dos colas), Z1- $\alpha / 2=1,645$ (valor tipificado), $\beta=0,3$ (máximo error tipo II), 1 - $\beta=0,7$ (poder estadístico), Z1- $\beta=0,524$ (valor tipificado), s12
$=7,44$ (varianza de dolor con paracetamol, según antecedente), s22 $=5,26$ (varianza de dolor de control positivo, según antecedente) y x1-x2=3,98 (diferencia propuesta), dando como resultado un mínimo de 18 pacientes por grupo.

Se incluyeron para el estudio a los pacientes ASA I de 18 a 45 años de edad, con grado de instrucción primaria completa e indicación de cirugía mucogingival, en estado asintomático. Se excluyeron del ensayo a los pacientes que no aceptaron participar, a aquellos en los que estaba contraindicada la cirugía oral, el uso de anestésico local (lidocaína) con epinefrina y/o la administración de los fármacos en estudio, con incapacidad intelectual y a gestantes o mujeres en período de lactancia y las que no utilizaban una forma adecuada de anticoncepción. Además se excluyeron a los pacientes que hubiesen recibido anestesia, sedantes, analgésicos, antidepresivos tricíclicos, corticosteroides, anticonvulsivantes, fenotiacinas, alcohol o cafeína 48 horas antes de la cirugía.

Una vez iniciado el estudio, la unidad muestral fue eliminada si no hubiese sido posible trabajar con el procedimiento estandarizado, si se excedió de dos cartuchos de anestesia para el procedimiento, si el paciente incumplió las indicaciones, si no llenó correctamente el instrumento, si no acudió a la cita control o si presentó alguna complicación postoperatoria.

Para la ejecución, se contó con la aprobación de la Escuela de Estomatología y de la Facultad de Medicina Humana de la Universidad Antenor Orrego. Se consideraron los principios éticos de la Declaración de Helsinki y de la Ley General de Salud del Perú (Ley No 26842).

Obtenidas las resoluciones correspondientes, se procedió a coordinar con los docentes responsables de las sesiones prácticas de la asignatura, además de solicitar el apoyo de los estudiantes. Previo al procedimiento se explicó al paciente la importancia del trabajo y, si aceptó participar voluntariamente, se le solicitó la lectura y firma del consentimiento informado.

El procedimiento estandarizado fue ejecutado por los estudiantes operadores de la asignatura, bajo supervisión de la investigadora principal.

Después de la antisepsia se utilizó benzocaína $20 \%$ como anestesia tópica en la zona de punción y 
lidocaína $2 \%$ con epinefrina 1:100000. Se usaron instrumentos similares para el procedimiento quirúrgico: mango de bisturí Bard Parker $\mathrm{N}^{\circ} 3$, hoja de bisturí $\mathrm{N}^{\circ}$ 15 y legra P24G. Para los casos de cirugía de reducción de bolsas se realizó colgajo de Widman modificado y para los casos de alargamiento de corona clínica, colgajo con desplazamiento apical. Se usaron además curetas Gracey Hu-Friedy de acuerdo a la zona intervenida y luego de concluida la cirugía, se suturó con hilo de seda negra 4/0.

Para el tratamiento analgésico, un colaborador independiente, distribuyó aleatoriamente a los pacientes de la siguiente manera:

- Grupo paracetamol: Se prescribió 500 mg de paracetamol vía oral, iniciando la primera toma 20 minutos después de finalizado el procedimiento y continuando cada 8 horas durante 24 horas.

- Grupo control: Se prescribió 400 mg de ibuprofeno vía oral, iniciando la primera toma 20 minutos después de finalizado el procedimiento y continuando cada 8 horas durante 24 horas.

Los pacientes recibieron el instrumento de medición y fueron capacitados para su correcto llenado, con las indicaciones correspondientes. Se instruyó a los pacientes para que cumplan con los requerimientos del estudio, pidiéndoseles que regresen a la cita de control (séptimo día) con el blíster del fármaco recetado y el instrumento de medición. Durante los días de estudio se prohibió el uso de antiácidos a base de óxido de magnesio o dióxido de aluminio, de las medicaciones esteroideas (a excepción de los anticonceptivos orales), y en general de toda medicación que pudiese confundir las evaluaciones. Se mantuvo contacto telefónico con el paciente, para monitorizar el cumplimiento de las indicaciones y posibles complicaciones. Si algún paciente hubiese considerado insuficiente el alivio de su dolor, se hubiera indicado un analgésico de rescate, analizándose el caso según el principio de intensión a tratar.

El instrumento de medición fue un cuestionario estructurado autoadministrado, donde se registró la intensidad de dolor en EVA $(0-100 \mathrm{~mm})$ a las $2,8 \mathrm{y}$ 24 horas posteriores a la toma inicial del medicamento. Los datos recolectados fueron procesados de manera automatizada en el programa estadístico SPSS Statistics versión 22.0 (IBM, Armonk, NY, USA), para luego presentar los resultados en tablas y/o gráficos estadísticos mostrando los resultados de acuerdo a los objetivos planteados. Se calcularon las medias, medianas, desviaciones estándar, valores mínimos y máximos. La comparación de la percepción del dolor entre paracetamol y el control positivo se realizó empleando la prueba U Mann-Whitney. Para la comparación general entre tiempos ( 2,8 y 24 horas) dentro de cada grupo en estudio se empleó la prueba de Friedman (k muestras pareadas) y Signo-Rango de Wilcoxon para las comparaciones 2 a 2 . Se consideró un nivel de significancia del $5 \%$.

\section{RESULTADOS}

Se tuvo como objetivo determinar la efectividad analgésica de paracetamol post-cirugías mucogingivales. La muestra estuvo constituida por 39 pacientes del curso de Periodoncia II que acudieron a la Clínica Estomatológica de la Universidad Privada Antenor Orrego en noviembre de 2016 y que cumplieron con los criterios establecidos. De estos, 19 recibieron paracetamol de $500 \mathrm{mg}$ y 20 recibieron ibuprofeno de $400 \mathrm{mg}$ (control). 2 pacientes fueron eliminados del estudio; el primero no cumplió con las indicaciones post-quirúrgicas y el segundo manifestó que paracetamol le ocasionó "aumento de la presión arterial“, y por eso dejó de tomarlo.

No existe diferencia en la efectividad analgésica entre paracetamol y el control post-cirugía mucogingival a las 2 horas $(p=0,415), 8$ horas $(p=0,279)$ y 24 horas $(p=0,736)$ (Tabla I).

Paracetamol es efectivo como analgésico postcirugía mucogingival a las 2,8 y 24 horas $(p<0.001)$ (Tabla II).

Ibuprofeno (control) es efectivo como analgésico post-cirugía mucogingival a las 2,8 y 24 horas $(p<$ 0,001) (Tabla III).

\section{DISCUSIÓN}

El dolor post-operatorio y edema después de un procedimiento quirúrgico periodontal es uno de los sucesos más comunes (Peres et al.). Por tal motivo, es necesario realizar la evaluación científica de fármacos que proporcionen a los pacientes una adecuada analgesia y comodidad para el período postoperatorio (Zardo et al.). 
Tabla I. Comparación de la efectividad analgésica de paracetamol post-cirugía mucogingival.

\begin{tabular}{llccccccc}
\hline $\begin{array}{l}\text { Efectividad } \\
\text { Analgésica }\end{array}$ & Analgésico & $\mathrm{N}$ & Media & Me & $\mathrm{DE}$ & Mín & Máx & \multirow{2}{*}{$\mathrm{p}^{*}$} \\
\hline \multirow{2}{*}{ a 2 hrs } & Paracetamol & 19 & 49,84 & 60,00 & 30,41 & 7 & 90 & \multirow{2}{*}{0,415} \\
& Control positivo & 20 & 42,95 & 45,00 & 29,21 & 7 & 87 & \\
a 8 hrs & Paracetamol & 19 & 37,53 & 42,00 & 23,81 & 6 & 88 & 0,279 \\
& Control positivo & 20 & 30,35 & 32,50 & 21,57 & 5 & 83 & \\
a 24 hrs & Paracetamol & 19 & 18,42 & 14,00 & 17,98 & 3 & 80 & \multirow{2}{*}{0,736} \\
& Control positivo & 20 & 15,65 & 11,50 & 11,28 & 5 & 47 & \\
\hline
\end{tabular}

*U de Mann-Whitney; DE, desviación estándar; Me, mediana; Mín, valor mínimo; Máx, valor máximo.

Tabla II. Eficacia analgésica de paracetamol post-cirugía mucogingival.

\begin{tabular}{lccccccc}
\hline $\begin{array}{l}\text { Efectividad } \\
\text { Analgésica }\end{array}$ & $\mathrm{n}$ & Media & Me & DE & Mín & Máx & $\mathrm{p}^{*}$ \\
\hline a 2 hrs (a) & 19 & $49,84^{\mathrm{b}, \mathrm{c}}$ & 60,00 & 30,41 & 7 & 90 & $<0,001$ \\
a 8 hrs (b) & 19 & $37,53^{\text {a,c }}$ & 42,00 & 23,81 & 6 & 88 & \\
a 24 hrs (c) & 19 & $18,42^{\text {a,b }}$ & 14,00 & 17,98 & 3 & 80 & \\
\hline
\end{tabular}

*Friedman; DE, desviación estándar; Me, mediana; Mín, valor mínimo; Máx, valor máximo. Los superíndices indican las letras con las cuales difieren estadísticamente (Wilcoxon $p<$ 0.05).

Tabla II. Eficacia analgésica de ibuprofeno post-cirugía mucogingival.

\begin{tabular}{|c|c|c|c|c|c|c|c|}
\hline $\begin{array}{l}\text { Efectividad } \\
\text { Analgésica }\end{array}$ & $\mathrm{n}$ & Media & $\mathrm{Me}$ & $\mathrm{DE}$ & Mín & Máx & $\mathrm{p}^{*}$ \\
\hline a 2 hrs (a) & 20 & $42,95 \mathrm{~b}^{\mathrm{c}}$ & 45,00 & 29,21 & 7 & 87 & \\
\hline a 8 hrs (b) & 20 & $30,35 a^{c}$ & 32,50 & 21,57 & 5 & 83 & $<0,001$ \\
\hline a $24 \mathrm{hrs}(\mathrm{c})$ & 20 & $15,65^{a, b}$ & 11,50 & 11,28 & 5 & 47 & \\
\hline
\end{tabular}

*Friedman; DE, desviación estándar; Me, mediana; Mín, valor mínimo; Máx, valor máximo. Los superíndices indican las letras con las cuales difieren estadísticamente (Wilcoxon $p<0.05)$.

Se carece de estudios que demuestren la eficacia de los fármacos para el control del dolor, después de una cirugía periodontal. Los resultados del presente trabajo indican que no existe diferencia entre paracetamol e ibuprofeno (control) a las 2, 8 y 24 horas.

Al realizar la búsqueda no se encontraron antecedentes. Sin embargo, En el 2009, Rashwan realizó un estudio piloto en El Cairo (Egipto), en donde se comparó paracetamol/cafeína vs ibuprofeno, llegando a la conclusión que paracetamol con cafeína, se puede usar de manera eficiente en el control del dolor postoperatorio después de cirugía a colgajo. Debido a la asociación de paracetamol con cafeína, el presente trabajo no puede ser comparado con el mencionado anteriormente.

En el presente estudio se evalúo la efectividad de paracetamol post cirugías de tratamiento de bolsas y alargamiento de corona. Paracetamol inhibe a las ciclooxigenasas, reduciendo la liberación de prostaglandinas en el sistema nervioso central, las concentraciones plasmáticas máximas se obtienen en 30 -
60 minutos (Coulthard et al.). Es un fármaco sumamente importante para el control del dolor, varios estudios han confirmado su efectividad, especialmente cuando los AINES están contraindicados (Deshpande et al., 2014).

Una limitante del presente trabajo que se debe mencionar es que las cirugías mucogingivales fueron realizadas por alumnos de pregrado, y debe considerarse las distintas habilidades psicomotrices de los operadores. Sin embargo, se espera que los criterios establecidos, así como, la asignación aleatoria de los grupos disminuya esta desventaja. Además se realizaron 2 tipos de cirugías mucogingivales, para reducción de bolsas y alargamientos de corona, debido a la cantidad limitada de casos.

Se requieren más estudios similares, con poblaciones mayores y homogéneas, que permitan continuar con la investigación de la efectividad analgésica de los fármacos para la práctica odontológica, en sus diferentes procedimientos. Es importante realizar ensa- 
yos clínicos con cada modelo de dolor y, según los resultados, innovar las alternativas farmacológicas, para cambiar la tradicional "prescripción basada en la experiencia" por la "prescripción basada en la evidencia".

\section{CONCLUSIONES}

Los resultados obtenidos permiten concluir que:

- No existe diferencia con respecto a la efectividad analgésica post-cirugía mucogingival entre paracetamol e ibuprofeno (control) a las 2,8 y 24 horas.

- Paracetamol es efectivo como analgésico post-cirugía mucogingival a las 2, 8 y 24 horas.

- Ibuprofeno (control) es efectivo como analgésico postcirugía mucogingival a las 2, 8 y 24 horas.

BURGOS QUIRÓZ, K. M.; ASMAT-ABANTO, A. S. \& ESPEJO-CARRERA, R. E. Analgesic effectiveness of paracetamol post-mucogingival surgery: Parallel randomized clinical trial. Int. J. Odontostomat., 13(2):184-188, 2019.

ABSTRACT: The objective of this study was to determine the analgesic effectiveness of paracetamol after mucogingival surgery. This prospective, longitudinal, comparative and experimental study was developed in the Stomatology Clinic of the Universidad Privada Antenor Orrego (Trujillo, Perú) with the patients of the Periodontics II subject. We included 39 patients, who were randomly distributed into 2 groups of 19 and 20 each, according to the established criteria. The medication was started 20 minutes after the surgery was completed, and was maintained for 24 hours. To determine the analgesic effectiveness, the analogue visual scale $(0-100 \mathrm{~mm})$ was used and the information that was processed was collected, using the $U$ Mann-Whitney test with a level of significance of 5 $\%$. There is no difference in terms of analgesic effectiveness post-mucogingival surgery between paracetamol and control at 2 hours $(p=0.415), 8$ hours $(p=0.279)$ and 24 hours $(p=$ 0.736 ). Paracetamol is effective as a mucogingival post-surgery analgesic.

KEY WORDS: effectiveness, analgesics, acetaminophen, periodontal, visual analog scale.

\section{REFERENCIAS BIBLIOGRÁFICAS}

Ardila Medina, C. M. Antiinflamatorios no esteroides como terapia adjunta al raspado y alisado radicular en periodontitis. Av. Periodoncia Implantol., 24(1):39-46, 2012.

Betancourt, J. W.; Kupp, L. I.; Jasper, S. J. \& Farooqi, O. A. Efficacy of ibuprofen-hydrocodone for the treatment of postoperative pain after periodontal surgery. J. Periodontal., 75(6):872-6, 2004.
Coulthard, P.; Bailey, E. \& Patel, N. Paracetamol (acetaminophen) for pain after oral surgery. Oral Surg., 7(2):81-6, 2014.

Deshpande, A.; Bhargava, D. \& Gupta, M. Analgesic efficacy of acetaminophen for controlling postextraction dental pain. Ann. Maxillofac. Surg., 4(2):176 -7, 2014.

Durand, R.; Tran, S. D.; Mui, B. \& Voyer, R. Managing postoperative pain following periodontal surgery. J. Can. Dent. Assoc., 79:d66, 2013.

Eli, L.; Baht, R.; Kozlovsky, A. \& Simon, H. Effect of gender on acute pain prediction and memory in periodontal surgery. Eur. J. Oral Sci., 108(2):99-103, 2000.

Gehling, M.; Arndt, C.; Eberhart, L. H.; Koch, T.; Krüger, T. \& Wulf, H. Postoperative analgesia with parecoxib, acetaminophen, and the combination of both: a randomized, double-blind, placebo-controlled trial in patients undergoing thyroid surgery. Br. J. Anaesth., 104(6):7617, 2010.

Godoy, C.; Guerrero, V. \& Lozano, E. Cirugía plástica periodontal, una realidad asistencial: reporte de casos. Rev. Clin. Periodoncia Implantol. Rehabil. Oral, 7(3):180-6, 2014.

Hungund, S. \& Thakkar, R. Effect of pretreatment with ketorolac tromethamine on operative pain during periodontal surgery: A casecontrol study. J. Indian Soc. Periodontol., 15(1):55-8, 2011.

Jaiswal, S. G.; Jaiswal, G. \& Gadbail, A. R. Assessment of postoperative pain of oral mucosal biopsy: A health initiative prospective study. Quintessence Int., 45(1):67-73, 2014.

Martínez Hernández, R. \& Marín González, M. G. Cirugía plástica periodontal en coronas clínicamente cortas para rehabilitación protésica. Rev. Odontol. Mex., 17(4):240-6, 2013.

Moore, R. A.; Straube, S.; Paine, J.; Derry, S. \& McQuay, H. J. Minimum efficacy criteria for comparisons between treatments using individual patient meta-analysis of acute pain trials: examples of etoricoxib, paracetamol, ibuprofen, and ibuprofen/paracetamol combinations after third molar extraction. Pain, 152(5):982-9, 2011.

Peres, M. F.; Ribeiro, F. V.; Ruiz, K. G.; Nociti, F. H. Jr.; Sallum, E. A. \& Casati, M. Z. Steroidal and non-steroidal cyclooxygenase-2 inhibitor anti-inflammatory drugs as pre-emptive medication in patients undergoing periodontal surgery. Braz. Dent. J., 23(6):621-8, 2012.

Pickett, F. A. Management of oral pain. World J. Dent., 3(2):207-12, 2012.

Ramachandran, A.; Khan, S. I.; Mohanavelu, D. \& Kumar, K. S. The efficacy of pre-operative oral medication of paracetamol, ibuprofen, and aceclofenac on the success of maxillary infiltration anesthesia in patients with irreversible pulpitis: A double-blind, randomized controlled clinical trial. J. Conserv. Dent., 15(4):310-4, 2012.

Rashwan, W. A. The efficacy of acetaminophen-caffeine compared to Ibuprofen in the control of postoperative pain after periodontal surgery: a crossover pilot study. J. Periodontol., 80(6):945-52, 2009.

Zahrowski, J. J. Good evidence supports acetaminophen as an effective and safe reliever of acute postoperative pain. J. Am. Dent. Assoc., 141(4):455-6, 2010.

Zardo, L. N.; dos Santos, F. A. \& Pilatti, G. L. Use of etoricoxib and dexamethasone for postoperative pain prevention and control in mucogingival surgery - a randomized parallel double-blind clinical trial. Braz. J. Oral Sci., 12(4):345-51, 2013.

Dirección para correspondencia:

Angel S. Asmat-Abanto

Doctor en Estomatología

Escuela de Postgrado de la Universidad Nacional de Trujillo

Docente de la Escuela Profesional de Estomatología

Universidad Privada Antenor Orrego

Trujillo

PERÚ

Email: aasmata1@upao.edu.pe

Recibido : 21-09-2018

Aceptado: 06-10-2018 